\title{
THE HOLOMORPHIC SECTIONAL CURVATURE OF GENERAL NATURAL KÄHLER STRUCTURES ON COTANGENT BUNDLES
}

\author{
BY \\ S.L. DRUŢA $\breve{A}^{*}$ \\ Dedicated to the memory of Professor Neculai Papaghiuc
}

\begin{abstract}
We study the conditions under which a Kählerian structure $(G, J)$ of general natural lift type on the cotangent bundle $T^{*} M$ of a Riemannian manifold $(M, g)$ has constant holomorphic sectional curvature. We obtain that a certain parameter involved in the condition for $\left(T^{*} M, G, J\right)$ to be a Kählerian manifold, is expressed as a rational function of the other two parameters, their derivatives, the constant sectional curvature of the base manifold $(M, g)$, and the constant holomorphic sectional curvature of the general natural Kählerian structure $(G, J)$.
\end{abstract}

Mathematics Subject Classification 2000: 53C55, 53C15, 53C07.

Key words: cotangent bundle, Riemannian metric, general natural lift.

1. Introduction. The natural lifts introduced on the cotangent bundle of a Riemannian manifold $(M, g)$ led to some geometric structures studied in the last years in a few papers like [9], [15]-[22]. A part of the results obtained in these works are similar to some results from the geometry of the tangent bundle $T M$, the dual of the cotangent bundle $T^{*} M$ (see [1], [2] [7],[10]-[12]). The differences which appear are related to the construction of the lifts on the cotangent bundle, the technics being different from those used in the geometry of the tangent bundle (see [23]).

In the paper [13], OpRoIU introduced the general expression for the natural 1-st order almost complex structure $J$ on the tangent bundle $T M$

\footnotetext{
*Partially supported by the Grant TD-158/2007, CNCSIS, Ministerul Educaţiei şi Cercetării, România
} 
and the notion of general natural lifted metric $G$ on $T M$, defined by the Riemannian metric $g$ from the base manifold, just like the natural lifts are obtained in [5] and [6]. With respect to the new metric the horizontal and vertical distributions are no more orthogonal to each other, contrary to the diagonal case, treated in [14]. The author obtained that the family of Kählerian structures $(G, J)$ of general natural lift type on $T M$ depends on three essential parameters (two of them are involved in the expression of the integrable almost complex structure $J$, and the third one is a certain proportionality factor, from the condition for $(G, J)$ to be almost Hermitian).

The present author defined in the paper [3], an almost complex structure of general natural lifted type on the cotangent bundle $T^{*} M$, and a general natural lifted metric to $T^{*} M$, obtained from the Riemannian metric $g$ of the base manifold $M$. The main result is that the family of general natural Kähler structures on $T^{*} M$ depends on three essential parameters (one is a certain proportionality factor obtained from the condition for the structure to be almost Hermitian and the other two are some coefficients involved in the definition of the integrable almost complex structure $J$ on $T^{*} M$ ).

In the joint work [4], Oproiu and the present author studied the conditions under which the Kählerian manifold $(T M, G, J)$ of general natural lift type has constant holomorphic sectional curvature. They obtained that the proportionality factor involved in the condition for $(T M, G, J)$ to be Kählerian is expressed as a rational function of the two essential parameters involved in the expression of $J$ (integrable almost complex structure on $T M)$, their derivatives, the constant sectional curvature of $(M, g)$ and the constant holomorphic sectional curvature of $(T M, G, J)$.

In the present paper we are interested in finding some properties of the curvature tensor field $K$ of the general natural Kähler structure $(G, J)$ on the cotangent bundle $T^{*} M$. Namely, we find the conditions under which the Kählerian structure considered on $T^{*} M$ has constant holomorphic sectional curvature. By doing some quite long computations with the RICCI package from Mathematica, we get the expressions of the components of the curvature tensor field of the manifold $\left(T^{*} M, G\right)$ and those of the curvature tensor field $K_{0}$ of the Kählerian manifold $\left(T^{*} M, G, J\right)$ having constant holomorphic sectional curvature $k$. The vanishing conditions for the components of the difference $K-K_{0}$ lead to the conclusion that $\left(T^{*} M, G, J\right)$ has constant holomorphic sectional curvature $k$, if and only if the proportionality factor involved in the condition for $\left(T^{*} M, G, J\right)$ to be Kählerian is a 
rational function depending on the two essential parameters involved in the expression of the integrable almost complex structure $J$, their derivatives, the constant sectional curvature of $(M, g)$ and $k$.

The manifolds, tensor fields and other geometric objects considered in this paper are assumed to be differentiable of class $C^{\infty}$ (i.e. smooth). The Einstein summation convention is used throughout this paper, the range of the indices $h, i, j, k, l, m, r$ being always $\{1, \ldots, n\}$.

2. Preliminary results. If $(M, g)$ is a smooth Riemannian manifold of the dimension $n$, and $\pi: T^{*} M \rightarrow M$ its cotangent bundle, then the total space $T^{*} M$ may be endowed with a structure of $2 n$-dimensional smooth manifold, induced from the structure of the base manifold, as follows: from every local chart $(U, \varphi)=\left(U, x^{1}, \ldots, x^{n}\right)$, it is induced a local chart, $\left(\pi^{-1}(U), \Phi\right)=\left(\pi^{-1}(U), q^{1}, \ldots, q^{n}, p_{1}, \ldots, p_{n}\right)$ on $T^{*} M$, such that for a cotangent vector $p \in \pi^{-1}(U) \subset T^{*} M$, the first $n$ local coordinates $q^{1}, \ldots, q^{n}$ are the local coordinates of its base point $x=\pi(p)$ in the local chart $(U, \varphi)$ (in fact we have $q^{i}=\pi^{*} x^{i}=x^{i} \circ \pi, i=1, \ldots n$ ); the last $n$ local coordinates $p_{1}, \ldots, p_{n}$ of $p \in \pi^{-1}(U)$ are the vector space coordinates of $p$ with respect to the natural basis $\left(d x_{\pi(p)}^{1}, \ldots, d x_{\pi(p)}^{n}\right)$, defined by the local chart $(U, \varphi)$, i.e. $p=p_{i} d x_{\pi(p)}^{i}$.

The notion of $M$-tensor field on the tangent bundle was introduced in the paper [8] and it is called also $d$-tensor field (e.g. see [2]). On the cotangent bundle $T^{*} M$, an $M$-tensor field of type $(r, s)$ is defined by sets of $n^{r+s}$ components (functions depending on $q^{i}$ and $p_{i}$ ), with $r$ upper indices and $s$ lower indices, assigned to the induced local charts $\left(\pi^{-1}(U), \Phi\right)$ on $T^{*} M$, such that the local coordinate change rule is that of the local coordinate components of a tensor field of type $(r, s)$ on the base manifold $M$. An usual tensor field of type $(r, s)$ on $M$ may be thought as an $M$-tensor field of type $(r, s)$ on $T^{*} M$. If the considered tensor field on $M$ is covariant only, the corresponding $M$-tensor field on $T^{*} M$ may be identified with the induced (pullback by $\pi$ ) tensor field on $T^{*} M$.

Some useful $M$-tensor fields on $T^{*} M$ may be obtained as follows. Let $v, w:[0, \infty) \rightarrow \mathbf{R}$ be smooth functions and let $\|p\|^{2}=g_{\pi(p)}^{-1}(p, p)$ be the square of the norm of the cotangent vector $p \in \pi^{-1}(U)\left(g^{-1}\right.$ is the tensor field of type $(2,0)$ having the components $\left(g^{k l}(x)\right)$ which are the entries of the inverse of the matrix $\left(g_{i j}(x)\right)$ defined by the components of $g$ in the local chart $(U, \varphi))$. The components $v\left(\|p\|^{2}\right) g_{i j}(\pi(p)), p_{i}, w\left(\|p\|^{2}\right) p_{i} p_{j}$ define respective $M$-tensor fields of types $(0,2),(0,1),(0,2)$ on $T^{*} M$. Similarly, 
the components $v\left(\|p\|^{2}\right) g^{k l}(\pi(p)), g^{0 i}=p_{h} g^{h i}, w\left(\|p\|^{2}\right) g^{0 k} g^{0 l}$ define respective $M$-tensor fields of type $(2,0),(1,0),(2,0)$ on $T^{*} M$. Of course, all the components considered above are in the induced local chart $\left(\pi^{-1}(U), \Phi\right)$.

We recall the splitting of the tangent bundle to $T^{*} M$ into the vertical distribution $V T^{*} M=\operatorname{Ker} \pi_{*}$ and the horizontal one determined by the Levi Civita connection $\dot{\nabla}$ of $g$ :

$$
T T^{*} M=V T^{*} M \oplus H T^{*} M .
$$

If $\left(\pi^{-1}(U), \Phi\right)=\left(\pi^{-1}(U), q^{1}, \ldots, q^{n}, p_{1}, \ldots, p_{n}\right)$ is a local chart on $T^{*} M$, induced from the local chart $(U, \varphi)=\left(U, x^{1}, \ldots, x^{n}\right)$, the local vector fields $\frac{\partial}{\partial p_{1}}, \ldots, \frac{\partial}{\partial p_{n}}$ on $\pi^{-1}(U)$ define a local frame for $V T^{*} M$ over $\pi^{-1}(U)$ and the local vector fields $\frac{\delta}{\delta q^{1}}, \ldots, \frac{\delta}{\delta q^{n}}$ define a local frame for $H T^{*} M$ over $\pi^{-1}(U)$, where

$$
\frac{\delta}{\delta q^{i}}=\frac{\partial}{\partial q^{i}}+\Gamma_{i h}^{0} \frac{\partial}{\partial p_{h}}, \quad \Gamma_{i h}^{0}=p_{k} \Gamma_{i h}^{k}
$$

and $\Gamma_{i h}^{k}(\pi(p))$ are the Christoffel symbols of $g$.

The set of vector fields $\left\{\frac{\partial}{\partial p_{1}}, \ldots, \frac{\partial}{\partial p_{n}}, \frac{\delta}{\delta q^{1}}, \ldots, \frac{\delta}{\delta q^{n}}\right\}$ defines a local frame on $T^{*} M$, adapted to the direct sum decomposition (1).

We consider

$$
t=\frac{1}{2}\|p\|^{2}=\frac{1}{2} g_{\pi(p)}^{-1}(p, p)=\frac{1}{2} g^{i k}(x) p_{i} p_{k}, \quad p \in \pi^{-1}(U)
$$

the energy density defined by $g$ in the cotangent vector $p$. We have $t \in[0, \infty)$ for all $p \in T^{*} M$.

The computations will be done in local coordinates, using a local chart $(U, \varphi)$ on $M$ and the induced local chart $\left(\pi^{-1}(U), \Phi\right)$ on $T^{*} M$.

We shall use the following lemma, which may be proved easily.

Lemma 2.1. If $n>1$ and $u, v$ are smooth functions on $T^{*} M$ such that

$$
u g_{i j}+v p_{i} p_{j}=0, \quad u g^{i j}+v g^{0 i} g^{0 j}=0, \quad \text { or } \quad u \delta_{j}^{i}+v g^{0 i} p_{j}=0,
$$

on the domain of any induced local chart on $T^{*} M$, then $u=0, v=0$.

In the paper [3], the present author considered the real valued smooth functions $a_{1}, a_{2}, a_{3}, a_{4}, b_{1}, b_{2}, b_{3}, b_{4}$ on $[0, \infty) \subset \mathbf{R}$ and studied a general natural tensor of type $(1,1)$ on $T^{*} M$, defined by the relations

$$
\left\{\begin{array}{c}
J X_{p}^{H}=a_{1}(t)\left(g_{X}\right)_{p}^{V}+b_{1}(t) p(X) p_{p}^{V}+a_{4}(t) X_{p}^{H}+b_{4}(t) p(X)\left(p^{\sharp}\right)_{p}^{H}, \\
J \theta_{p}^{V}=a_{3}(t) \theta_{p}^{V}+b_{3}(t) g_{\pi(p)}^{-1}(p, \theta) p_{p}^{V}-a_{2}(t)\left(\theta^{\sharp}\right)_{p}^{H} \\
\quad-b_{2}(t) g_{\pi(p)}^{-1}(p, \theta)\left(p^{\sharp}\right)_{p}^{H},
\end{array}\right.
$$


in every point $p$ of the induced local card $\left(\pi^{-1}(U), \Phi\right)$ on $T^{*} M, \forall X \in$ $\mathcal{X}(M), \forall \theta \in \Lambda^{1}(M)$, where $g_{X}$ is the 1 -form on $M$ defined by $g_{X}(Y)=$ $g(X, Y), \forall Y \in \mathcal{X}(M), \theta^{\sharp}=g_{\theta}^{-1}$ is a vector field on $M$ defined by $g\left(\theta^{\sharp}, Y\right)=$ $\theta(Y), \forall Y \in \mathcal{X}(M)$, the vector $p^{\sharp}$ is tangent to $M$ in $\pi(p), p^{V}$ is the Liouville vector field on $T^{*} M$, and $\left(p^{\sharp}\right)^{H}$ is the similar horizontal vector field on $T^{*} M$.

With respect to the adapted frame $\left\{\frac{\partial}{\partial p_{i}}, \frac{\delta}{\delta q^{j}}\right\}_{i, j=1, \ldots, n}$ on $T^{*} M$, the expression (3) becomes

$$
\left\{\begin{array}{l}
J \frac{\delta}{\delta q^{i}}=a_{1}(t) g_{i j} \frac{\partial}{\partial p_{j}}+b_{1}(t) p_{i} C+a_{4}(t) \frac{\delta}{\delta q^{i}}+b_{4}(t) p_{i} \widetilde{C} \\
J \frac{\partial}{\partial p_{i}}=a_{3}(t) \frac{\partial}{\partial p_{i}}+b_{3}(t) g^{0 i} C-a_{2}(t) g^{i j} \frac{\delta}{\delta q^{j}}-b_{2}(t) g^{0 i} \widetilde{C}
\end{array}\right.
$$

where $C=p^{V}$ is the Liouville vector-field on $T^{*} M$ and $\widetilde{C}=\left(p^{\sharp}\right)^{H}$ is the corresponding horizontal vector field on $T^{*} M$.

We may write also

$$
\left\{\begin{array}{l}
J \frac{\delta}{\delta q^{i}}=J_{i j}^{(1)} \frac{\partial}{\partial p_{j}}+J 4_{i}^{j} \frac{\delta}{\delta q^{j}} \\
J \frac{\partial}{\partial p_{i}}=J 3_{j}^{i} \frac{\partial}{\partial p_{j}}-J_{(2)}^{i j} \frac{\delta}{\delta q^{j}}
\end{array}\right.
$$

where

$$
\begin{aligned}
& J_{i j}^{(1)}=a_{1}(t) g_{i j}+b_{1}(t) p_{i} p_{j}, \quad J 4_{i}^{j}=a_{4}(t) \delta_{i}^{j}+b_{4}(t) g^{0 j} p_{i} \\
& J 3_{j}^{i}=a_{3}(t) \delta_{j}^{i}+b_{3}(t) g^{0 i} p_{j}, \quad J_{(2)}^{i j}=a_{2}(t) g^{i j}+b_{2}(t) g^{0 i} g^{0 j} .
\end{aligned}
$$

Theorem $2.2([3])$. A natural tensor field $J$ of type $(1,1)$ on $T^{*} M$ given by (4) or (5) defines an almost complex structure on $T^{*} M$, if and only if $a_{4}=-a_{3}, b_{4}=-b_{3}$ and the coefficients $a_{1}, a_{2}, a_{3}, b_{1}, b_{2}$ and $b_{3}$ are related by

$$
a_{1} a_{2}=1+a_{3}^{2}, \quad\left(a_{1}+2 t b_{1}\right)\left(a_{2}+2 t b_{2}\right)=1+\left(a_{3}+2 t b_{3}\right)^{2} .
$$

Remark. From the conditions (6) we have that the coefficients $a_{1}, a_{2}, a_{1}+$ $2 t b_{1}, a_{2}+2 t b_{2}$ have the same sign and maynot vanish. We assume that $a_{1}>0, a_{2}>0, a_{1}+2 t b_{1}>0, a_{2}+2 t b_{2}>0$ for all $t \geq 0$. 
Remark. The relations (6) allow us to express two of the coefficients $a_{1}, a_{2}, a_{3}, b_{1}, b_{2}, b_{3}$ as functions of the other four; e.g. we have:

$$
a_{2}=\frac{1+a_{3}^{2}}{a_{1}}, \quad b_{2}=\frac{2 a_{3} b_{3}-a_{2} b_{1}+2 t b_{3}^{2}}{a_{1}+2 t b_{1}} .
$$

The integrability condition for the above almost complex structure $J$ on a manifold $M$ is characterized by the vanishing of its Nijenhuis tensor field $N_{J}$, defined by

$$
N_{J}(X, Y)=[J X, J Y]-J[J X, Y]-J[X, J Y]-[X, Y],
$$

for all the vector fields $X$ and $Y$ on $M$.

Theorem 2.3 ([3]). Let $(M, g)$ be an $n(>2)$-dimensional connected Riemannian manifold. The almost complex structure J defined by (4) on $T^{*} M$ is integrable if and only if $(M, g)$ has constant sectional curvature $c$ and the coefficients $b_{1}, b_{2}, b_{3}$ are given by:

$$
\left\{\begin{array}{l}
b_{1}=\frac{2 c^{2} t a_{2}^{2}+2 c t a_{1} a_{2}^{\prime}+a_{1} a_{1}^{\prime}-c+3 c a_{3}^{2}}{a_{1}-2 t a_{1}^{\prime}-2 c t a_{2}-4 c t^{2} a_{2}^{\prime}} \\
b_{2}=\frac{2 t a_{3}^{\prime 2}-2 t a_{1}^{\prime} a_{2}^{\prime}+c a_{2}^{2}+2 c t a_{2} a_{2}^{\prime}+a_{1} a_{2}^{\prime}}{a_{1}-2 t a_{1}^{\prime}-2 c t a_{2}-4 c t^{2} a_{2}^{\prime}} \\
b_{3}=\frac{a_{1} a_{3}^{\prime}+2 c a_{2} a_{3}+4 c t a_{2}^{\prime} a_{3}-2 c t a_{2} a_{3}^{\prime}}{a_{1}-2 t a_{1}^{\prime}-2 c t a_{2}-4 c t^{2} a_{2}^{\prime}}
\end{array}\right.
$$

Remark. In the diagonal case, where $a_{3}=0$ it follows $b_{3}=0$ too, and we have:

$$
a_{2}=\frac{1}{a_{1}}, b_{1}=\frac{a_{1} a_{1}^{\prime}-c}{a_{1}-2 t a_{1}^{\prime}}, b_{2}=\frac{c-a_{1} a_{1}^{\prime}}{a_{1}\left(a_{1}^{2}-2 c t\right)} .
$$

In the paper cited bellow, the present author introduced a Riemannian metric $G$ of general natural lift type on $T^{*} M$, defined by the relations

$$
\left\{\begin{array}{l}
G_{p}\left(X^{H}, Y^{H}\right)=c_{1}(t) g_{\pi(p)}(X, Y)+d_{1}(t) p(X) p(Y) \\
G_{p}\left(\theta^{V}, \omega^{V}\right)=c_{2}(t) g_{\pi(p)}^{-1}(\theta, \omega)+d_{2}(t) g_{\pi(p)}^{-1}(p, \theta) g_{\pi(p)}^{-1}(p, \omega) \\
G_{p}\left(X^{H}, \theta^{V}\right)=G_{p}\left(\theta^{V}, X^{H}\right)=c_{3}(t) \theta(X)+d_{3}(t) p(X) g_{\pi(p)}^{-1}(p, \theta),
\end{array}\right.
$$


$\forall X, Y \in \mathcal{X}(M), \forall \theta, \omega \in \Lambda^{1}(M), \forall p \in T^{*} M$.

Using the adapted frame $\left\{\frac{\partial}{\partial p_{i}}, \frac{\delta}{\delta q^{j}}\right\}_{i, j=1, \ldots, n}$ on $T^{*} M$, we may write the expression (9) in the next form

$$
\left\{\begin{array}{l}
G\left(\frac{\delta}{\delta q^{i}}, \frac{\delta}{\delta q^{j}}\right)=c_{1}(t) g_{i j}+d_{1}(t) p_{i} p_{j}=G_{i j}^{(1)} \\
G\left(\frac{\partial}{\partial p_{i}}, \frac{\partial}{\partial p_{j}}\right)=c_{2}(t) g^{i j}+d_{2}(t) g^{0 i} g^{0 j}=G_{(2)}^{i j}, \\
G\left(\frac{\partial}{\partial p_{i}}, \frac{\delta}{\delta q^{j}}\right)=G\left(\frac{\delta}{\delta q^{i}}, \frac{\partial}{\partial p_{j}}\right)=c_{3}(t) \delta_{i}^{j}+d_{3}(t) p_{i} g^{0 j}=G 3_{i}^{j}
\end{array}\right.
$$

where $c_{1}, c_{2}, c_{3}, d_{1}, d_{2}, d_{3}$ are six smooth functions of the density energy on $T^{*} M$.

The conditions for $G$ to be positive definite are assured if

(11) $c_{1}+2 t d_{1}>0, c_{2}+2 t d_{2}>0,\left(c_{1}+2 t d_{1}\right)\left(c_{2}+2 t d_{2}\right)-\left(c_{3}+2 t d_{3}\right)^{2}>0$.

The metric $G$ is almost Hermitian with respect to the general almost complex structure $J$, if $G(J X, J Y)=G(X, Y)$, for all the vector fields $X, Y$ on $T^{*} M$.

The author proved the following result

Theorem 2.4 ([3]). The family of general natural Riemannian metrics $G$ on $T^{*} M$ such that $\left(T^{*} M, G, J\right)$ is an almost Hermitian manifold, is given by (10), provided that the coefficients $c_{1}, c_{2}, c_{3}, d_{1}, d_{2}$, and $d_{3}$ are related to the coefficients $a_{1}, a_{2}, a_{3}, b_{1}, b_{2}$, and $b_{3}$ by the following proportionality relations

$$
\begin{gathered}
\frac{c_{1}}{a_{1}}=\frac{c_{2}}{a_{2}}=\frac{c_{3}}{a_{3}}=\lambda \\
\frac{c_{1}+2 t d_{1}}{a_{1}+2 t b_{1}}=\frac{c_{2}+2 t d_{2}}{a_{2}+2 t b_{2}}=\frac{c_{3}+2 t d_{3}}{a_{3}+2 t b_{3}}=\lambda+2 t \mu,
\end{gathered}
$$

where the proportionality coefficients $\lambda>0$ and $\lambda+2 t \mu>0$ are some functions depending on $t$.

Remark. In the case where $a_{3}=0$, it follows that $c_{3}=d_{3}=0$ and we obtain the almost Hermitian structure considered in [18], [20]. Moreover, if 
$\lambda=1$ and $\mu=0$, we obtain the almost Kählerian structure considered in the mentioned papers.

Considering the two-form $\Omega$ defined by the almost Hermitian structure $(G, J)$ on $T^{*} M$

$$
\Omega(X, Y)=G(X, J Y),
$$

for all the vector fields $X, Y$ on $T^{*} M$, we obtain the following result from [3]:

Proposition 2.5 ([3]). The expression of the 2 -form $\Omega$ in the local adapted frame $\left\{\frac{\partial}{\partial p_{i}}, \frac{\delta}{\delta q^{j}}\right\}_{i, j=1, \ldots, n}$ on $T^{*} M$, is given by

$$
\Omega\left(\frac{\partial}{\partial p_{i}}, \frac{\partial}{\partial p_{j}}\right)=0, \Omega\left(\frac{\delta}{\delta q^{i}}, \frac{\delta}{\delta q^{j}}\right)=0, \Omega\left(\frac{\partial}{\partial p_{i}}, \frac{\delta}{\delta q^{j}}\right)=\lambda \delta_{j}^{i}+\mu g^{0 i} p_{j}
$$

or, equivalently

$$
\Omega=\left(\lambda \delta_{j}^{i}+\mu g^{0 i} p_{j}\right) D p_{i} \wedge d q^{j},
$$

where $D p_{i}=d p_{i}-\Gamma_{i h}^{0} d q^{h}$ is the absolute differential of $p_{i}$.

Next, by calculating the exterior differential of $\Omega$, we may state:

Theorem 2.6 ([3]). The almost Hermitian structure $\left(T^{*} M, G, J\right)$ is almost Kählerian if and only if $\mu=\lambda^{\prime}$.

Remark. The family of general natural almost Kählerian structures on $T^{*} M$ depends on five essential coefficients $a_{1}, a_{3}, b_{1}, b_{3}, \lambda$, which must satisfy the supplementary conditions $a_{1}>0, a_{1}+2 t b_{1}>0, \lambda>0, \lambda+2 t \mu>$ 0 .

The main result obtained in [3] is the next one:

Theorem 2.7 ([3]). A general natural lifted almost Hermitian structure $(G, J)$ on $T^{*} M$ is Kählerian if and only if the almost complex structure $J$ is integrable (see Theorem 2.3) and $\mu=\lambda^{\prime}$.

Remark. The family of general natural Kählerian structures on $T^{*} M$ depends on three essential coefficients $a_{1}, a_{3}, \lambda$, which must satisfy the supplementary conditions $a_{1}>0, a_{1}+2 t b_{1}>0, \lambda>0, \lambda+2 t \lambda^{\prime}>0$, where $b_{1}$ is is given by (8).

Examples of such structures may be found in [18], [20]. 
3. General natural Kähler structures of constant holomorphic sectional curvature on cotangent bundles. The Levi-Civita connection $\nabla$ of the Riemannian manifold $\left(T^{*} M, G\right)$ is obtained from the formula

$$
\begin{aligned}
2 G\left(\nabla_{X} Y, Z\right) & =X(G(Y, Z))+Y(G(X, Z))-Z(G(X, Y)) \\
& +G([X, Y], Z)-G([X, Z], Y)-G([Y, Z], X) ; \forall X, Y, Z \in \chi(M)
\end{aligned}
$$

and it is characterized by the conditions $\nabla G=0, T=0$, where $T$ is the torsion tensor of $\nabla$.

In the case of the cotangent bundle $T^{*} M$ we may obtain the explicit expression of $\nabla$. The symmetric $2 n \times 2 n$ matrix

$$
\left(\begin{array}{ll}
G_{i j}^{(1)} & G 3_{i}^{j} \\
G 3_{j}^{i} & G_{(2)}^{i j}
\end{array}\right)
$$

associated to the metric $G$ in the base $\left\{\frac{\delta}{\delta q^{i}}, \frac{\partial}{\partial p_{j}}\right\}_{i, j=1, \ldots, n}$ has the inverse

$$
\left(\begin{array}{ll}
H_{(1)}^{i j} & H 3_{i}^{j} \\
H 3_{j}^{i} & H_{i j}^{(2)}
\end{array}\right)
$$

where the entries are the blocks

(15) $H_{(1)}^{k l}=e_{1} g^{k l}+f_{1} g^{0 k} g^{0 l}, H_{k l}^{(2)}=e_{2} g_{k l}+f_{2} p_{k} p_{l}, H 3_{l}^{k}=e_{3} \delta_{l}^{k}+f_{3} g^{0 k} p_{l}$.

Here $g^{k l}$ are the components of the inverse of the matrix $\left(g_{i j}\right), g^{0 k}=p_{i} g^{i k}$, and $e_{1}, f_{1}, e_{2}, f_{2}, e_{3}, f_{3}:[0, \infty) \rightarrow \mathbb{R}$, some real smooth functions. Their expressions are obtained by solving the system:

$$
\left\{\begin{array}{l}
G_{i h}^{(1)} H_{(1)}^{h k}+G 3_{i}^{h} H 3_{h}^{k}=\delta_{i}^{k} \\
G_{i h}^{(1)} H 3_{k}^{h}+G 3_{i}^{h} H_{h k}^{(2)}=0 \\
G 3_{h}^{i} H H_{(1)}^{h k}+G_{(2)}^{i h} H 3_{h}^{k}=0 \\
G 3_{h}^{i} H 3_{k}^{h}+G_{(2)}^{i h} H_{h k}^{(2)}=\delta_{k}^{i},
\end{array}\right.
$$

in which we substitute the relations (10) and (15). By using lemma 2.1, we get $e_{1}, e_{2}, e_{3}$ as functions of $c_{1}, c_{2}, c_{3}$

$$
e_{1}=\frac{c_{2}}{c_{1} c_{2}-c_{3}^{2}}, \quad e_{2}=\frac{c_{1}}{c_{1} c_{2}-c_{3}^{2}}, \quad e_{3}=-\frac{c_{3}}{c_{1} c_{2}-c_{3}^{2}}
$$


and $f_{1}, f_{2}, f_{3}$ as functions of $c_{1}, c_{2}, c_{3}, d_{1}, d_{2}, d_{3}, e_{1}, e_{2}, e_{3}$

$$
\begin{aligned}
f_{1}= & -\frac{c_{2} d_{1} e_{1}-c_{3} d_{3} e_{1}-c_{3} d_{2} e_{3}+c_{2} d_{3} e_{3}+2 d_{1} d_{2} e_{1} t-2 d_{3}^{2} e_{1} t}{c_{1} c_{2}-c_{3}^{2}+2 c_{2} d_{1} t+2 c_{1} d_{2} t-4 c_{3} d_{3} t+4 d_{1} d_{2} t^{2}-4 d_{3}^{2} t^{2}}, \\
f_{2}= & \frac{\left(c_{3}+2 d_{3} t\right)\left[\left(d_{3} e_{1}+d_{2} e_{3}\right)\left(c_{1}+2 d_{1} t\right)-\left(d_{1} e_{1}+d_{3} e_{3}\right)\left(c_{3}+2 d_{3} t\right)\right]}{\left(c_{2}+2 d_{2} t\right)\left[\left(c_{1}+2 d_{1} t\right)\left(c_{2}+2 d_{2} t\right)-\left(c_{3}+2 d_{3} t\right)^{2}\right]} \\
& -\frac{d_{2} e_{2}+d_{3} e_{3}}{c_{2}+2 d_{2} t} \\
f_{3}= & -\frac{\left(d_{3} e_{1}+d_{2} e_{3}\right)\left(c_{1}+2 d_{1} t\right)-\left(d_{1} e_{1}+d_{3} e_{3}\right)\left(c_{3}+2 d_{3} t\right)}{\left(c_{1}+2 d_{1} t\right)\left(c_{2}+2 d_{2} t\right)-\left(c_{3}+2 d_{3} t\right)^{2}} .
\end{aligned}
$$

Next we may obtain the expression of the Levi Civita connection of the Riemannian metric $G$ on $T^{*} M$.

Theorem 3.1. The Levi-Civita connection $\nabla$ of $G$ has the following expression in the local adapted frame $\left\{\frac{\delta}{\delta q^{i}}, \frac{\partial}{\partial p_{j}}\right\}_{i, j=1, \ldots, n}$

$$
\left\{\begin{array}{l}
\nabla_{\frac{\partial}{\partial p_{i}}} \frac{\partial}{\partial p_{j}}=Q^{i j}{ }_{h} \frac{\partial}{\partial p_{h}}+\widetilde{Q}^{i j h} \frac{\delta}{\delta q^{h}}, \\
\nabla_{\frac{\delta}{\delta q^{i}}} \frac{\partial}{\partial p_{j}}=\left(-\Gamma_{i h}^{j}+\widetilde{P}_{i}{ }^{j}{ }_{h}\right) \frac{\partial}{\partial p_{h}}+P_{i}{ }^{j h} \frac{\delta}{\delta q^{h}} \\
\nabla_{\frac{\partial}{\partial p_{i}}} \frac{\delta}{\delta q^{j}}=P_{j}{ }^{i h} \frac{\delta}{\delta q^{h}}+\widetilde{P}_{j}{ }^{i} h \frac{\partial}{\partial p_{h}}, \\
\nabla_{\frac{\delta}{\delta q^{i}}} \frac{\delta}{\delta q^{j}}=\left(\Gamma_{i j}^{h}+\widetilde{S}_{i j}{ }^{h}\right) \frac{\delta}{\delta p_{h}}+S_{i j h} \frac{\partial}{\partial p_{h}},
\end{array}\right.
$$

where $\Gamma_{i j}^{h}$ are the Christoffel symbols of the connection $\dot{\nabla}$ and the $M$-tensor fields appearing as coefficients in the above expressions are given by

$$
\left\{\begin{array}{l}
Q^{i j}{ }_{h}=\frac{1}{2}\left(\partial^{i} G_{(2)}^{j k}+\partial^{j} G_{(2)}^{i k}-\partial^{k} G_{(2)}^{i j}\right) H_{k h}^{(2)}+\frac{1}{2}\left(\partial^{i} G 3_{k}^{j}+\partial^{j} G 3_{k}^{i}\right) H 3_{h}^{k} \\
\widetilde{Q}^{i j h}=\frac{1}{2}\left(\partial^{i} G_{(2)}^{j k}+\partial^{j} G_{(2)}^{i k}-\partial^{k} G_{(2)}^{i j}\right) H 3_{k}^{h}+\frac{1}{2}\left(\partial^{i} G 3_{k}^{j}+\partial^{j} G 3_{k}^{i}\right) H_{(1)}^{k h} \\
P_{j}{ }^{i h}=\frac{1}{2}\left(\partial^{i} G 3_{j}^{k}-\partial^{k} G 3_{j}^{i}\right) H 3_{k}^{h}+\frac{1}{2}\left(\partial^{i} G_{j k}^{(1)}-R_{l j k}^{0} G_{(2)}^{l i}\right) H_{(1)}^{k h}, \\
\widetilde{P}_{j h}{ }^{i}=\frac{1}{2}\left(\partial^{i} G 3_{j}^{k}-\partial^{k} G 3_{j}^{i}\right) H_{k h}^{(2)}+\frac{1}{2}\left(\partial^{i} G_{j k}^{(1)}-R_{l j k}^{0} G_{(2)}^{l i}\right) H 3_{h}^{k} \\
S_{i j h}=\frac{1}{2}\left(R_{l i j}^{0} G_{(2)}^{l k}-\partial^{k} G_{i j}^{(1)}\right) H_{k h}^{(2)}-c_{3} R_{i j k}^{0} H 3_{h}^{k}, \\
\widetilde{S}_{i j}{ }^{h}=\frac{1}{2}\left(R_{l i j}^{0} G_{(2)}^{l k}-\partial^{k} G_{i j}^{(1)}\right) H 3_{k}^{h}-c_{3} R_{i j k}^{0} H_{(1)}^{k h}
\end{array}\right.
$$

where $R_{k i j}^{h}$ are the components of the curvature tensor field of the Levi Civita connection $\dot{\nabla}$ of the base manifold $(M, g)$. 
If we replace in (19) the relations (10) which define the metric $G$, the expressions (15) for the inverse matrix $H$ of $G$, and the formulas (17), (18) we obtain the detailed expressions of $P_{i}{ }^{j h}, Q^{i j}{ }_{h}, S_{i j h}, \widetilde{P}_{j h}{ }^{i}, \widetilde{Q}^{i j h}, \widetilde{S}_{i j}{ }^{h}$.

The curvature tensor field $K$ of the connection $\nabla$ is defined by

$$
K(X, Y) Z=\nabla_{X} \nabla_{Y} Z-\nabla_{Y} \nabla_{X} Z-\nabla_{[X, Y]} Z, \quad X, Y, Z \in \mathcal{X}(T M) .
$$

By using the local adapted frame $\left\{\frac{\delta}{\delta q^{i}}, \frac{\partial}{\partial p_{j}}\right\}_{i, j=1, \ldots, n}=\left\{\delta_{i}, \partial^{j}\right\}_{i, j=1, \ldots, n}$ we obtain the horizontal and vertical components of the curvature tensor field:

$$
\begin{aligned}
K\left(\delta_{i}, \delta_{j}\right) \delta_{k} & =Q Q Q Q_{i j k}{ }^{h} \delta_{h}+Q Q Q P_{i j k h} \partial^{h}, \\
K\left(\delta_{i}, \delta_{j}\right) \partial^{k} & =Q Q P Q_{i j}{ }^{k h} \delta_{h}+Q Q P P_{i j}{ }_{h} \partial^{h}, \\
K\left(\partial^{i}, \partial^{j}\right) \delta_{k} & =P P Q Q^{i j}{ }_{k}{ }^{h} \delta_{h}+P P Q P^{i j}{ }_{k h} \partial^{h}, \\
K\left(\partial^{i}, \partial^{j}\right) \partial^{k} & =P P P Q^{i j k h} \delta_{h}+P P P P^{i j k}{ }_{h} \partial^{h}, \\
K\left(\partial^{i}, \delta_{j}\right) \delta_{k} & =P Q Q Q^{i}{ }_{j k}{ }^{h} \delta_{h}+P Q Q P^{i}{ }_{j k h} \partial^{h}, \\
K\left(\partial^{i}, \delta_{j}\right) \partial^{k} & =P Q P Q^{i}{ }_{j}{ }^{k h} \delta_{h}+P Q P P_{j}^{i}{ }_{h} \partial^{h},
\end{aligned}
$$

where the coefficients are the $M$-tensor fields given by

$$
\begin{aligned}
& Q Q Q Q_{i j k}^{h}=\widetilde{S}_{j k}^{l} \widetilde{S}_{i l}^{h}+P_{i}^{l h} S_{j k l}-\widetilde{S}_{j l}{ }^{h} \widetilde{S}_{i k}^{l}-P_{j}^{l h} S_{i k l} \\
& -R_{l i j}^{0} P_{k}^{l h}+R_{k i j}^{h} \\
& Q Q Q P_{i j k h}=\widetilde{S}_{j k}^{l} S_{i l h}+\widetilde{P}_{i h}^{l} S_{j k l}-\widetilde{S}_{i k}^{l} S_{j l h}-\widetilde{P}_{j h}^{l} S_{i k l}-\widetilde{P}_{k h}^{l} R_{l i j}^{0} \\
& Q Q P Q_{i j}{ }^{k h}=\widetilde{P}_{j}{ }^{k}{ }_{l} P_{i}{ }^{l h}+P_{j}{ }^{k l} \widetilde{S}_{i l}{ }^{h}-\widetilde{P}_{i}{ }^{k}{ }_{l} P_{j}{ }^{l h}-P_{i}{ }^{k l} \widetilde{S}_{j l}{ }^{h} \\
& -R_{l i j}^{0} \widetilde{Q}^{l k h} \\
& Q Q P P_{i j}{ }^{k}{ }_{h} \partial^{h}=\widetilde{P}_{j}{ }^{k}{ }_{l} \widetilde{P}_{i}{ }^{l}{ }_{h}+P_{j}{ }^{k l} S_{i l h}-\widetilde{P}_{i}{ }^{k}{ }_{l} \widetilde{P}_{j h}{ }^{l}-P_{i}{ }^{k l} S_{j l h} \\
& -R_{l i j}^{0} Q_{h}^{l k}-R_{l i j}^{k} \\
& P P Q Q^{i j}{ }_{k}^{h} \delta_{h}=\partial^{i} P_{k}{ }^{j h}-\partial^{j} P_{k}{ }^{i h}+\widetilde{P}_{k}{ }^{j}{ }_{l} \widetilde{Q}^{i l h}+P_{k}{ }^{j l} P_{l}{ }^{i h} \\
& -\widetilde{P}_{k}{ }_{l} \widetilde{Q}^{j l h}-P_{k}{ }^{i l} P_{l}{ }^{j h}, \\
& P P Q P^{i j}{ }_{k h}=\partial^{i} \widetilde{P}_{k h}^{j}-\partial^{j} \widetilde{P}_{k h}{ }^{i}+\widetilde{P}_{k l}{ }_{l} Q^{i l}{ }_{h}+P_{k}{ }^{j l} \widetilde{P}_{l h}{ }^{i} \\
& -\widetilde{P}_{k l}^{i} Q_{h}^{j l}-P_{k}^{i l} \widetilde{P}_{l}{ }^{j}{ }_{h}, \\
& P P P Q^{i j k h}=\partial^{i} \widetilde{Q}^{j k h}-\partial^{j} \widetilde{Q}^{i k h}+Q^{j k} \widetilde{Q}^{i l h}+\widetilde{Q}^{j k l} P_{l}{ }^{i h} \\
& -Q^{i k}{ }_{l} \widetilde{Q}^{j l h}-\widetilde{Q}^{i k l} P_{l}{ }^{j h},
\end{aligned}
$$




$$
\begin{aligned}
& P P P P^{i j k}{ }_{h}=\partial^{i} Q_{h}^{j k}-\partial^{j} Q^{i k}{ }_{h}+Q^{j k}{ }_{l} Q^{i l}{ }_{h}+\widetilde{Q}^{j k l} \widetilde{P}_{l}{ }^{i}{ }_{h} \\
& -Q^{i k}{ }_{l} Q_{h}^{j l}-\widetilde{Q}^{i k l} \widetilde{P}_{l}{ }^{j}{ }_{h}, \\
& P Q Q Q^{i}{ }_{j k}^{h} \delta_{h}=\partial^{i} \widetilde{S}_{j k}^{h}+S_{j k l} \widetilde{Q}^{i l h}+\widetilde{S}_{j k}^{l} P_{l}^{i h}-\widetilde{P}_{k l}^{i} P_{j}^{l h}-P_{k}^{i l} \widetilde{S}_{j l}^{h} \\
& P Q Q P^{i}{ }_{j k h}=\partial^{i} S_{j k h}+\widetilde{S}_{j k}^{l} Q_{h}^{i l}+\widetilde{S}_{j k}{ }^{l} \widetilde{P}_{l h}^{i}-\widetilde{P}_{k l}^{i} \widetilde{P}_{j h}^{l}-P_{k}^{i l} S_{j l h} \\
& P Q P Q^{i}{ }_{j}{ }^{k h}=\partial^{i} P_{j}{ }^{k h}+\widetilde{P}_{j}{ }_{l} \widetilde{Q}^{i l h}+P_{j}{ }^{k l} P_{l}{ }^{i h}-Q^{i k}{ }_{l} P_{j}{ }^{l h}-\widetilde{Q}^{i k l} \widetilde{S}_{j l}{ }^{h} \text {, } \\
& P Q P P_{j}^{i}{ }_{h}^{k}=\partial^{i} \widetilde{P}_{j h}^{k}+\widetilde{P}_{j}{ }_{l}^{k} Q^{i l}{ }_{h}+P_{j}{ }^{k l} \widetilde{P}_{l}{ }_{h}^{i}-Q^{i k}{ }_{l} \widetilde{P}_{j h}^{l}-\widetilde{Q}^{i k l} S_{j l h} .
\end{aligned}
$$

In order to get the final expressions of the above $M$-tensor fields, we have to compute the first and second order partial derivatives with respect to the cotangential coordinates $p_{i}$ of the usual tensor fields involved in the definition of the Riemannian metric $G$.

$$
\begin{aligned}
\partial^{i} G_{j k}^{(1)} & =c_{1}^{\prime} g^{0 i} g_{j k}+d_{1}^{\prime} g^{0 i} p_{j} p_{k}+d_{1} \delta_{j}^{i} p_{k}+d_{1} p_{j} \delta_{k}^{i} \\
\partial^{i} G_{(2)}^{j k} & =c_{2}^{\prime} g^{0 i} g^{j k}+d_{2}^{\prime} g^{0 i} g^{0 j} g^{0 k}+d_{2} g^{i j} g^{0 k}+d_{2} g^{0 j} g^{i k} \\
\partial^{i} G 3_{k}^{j} & =c_{3}^{\prime} g^{0 i} \delta_{k}^{j}+d_{3}^{\prime} g^{0 i} g^{0 j} p_{k}+d_{3} g^{i j} p_{k}+d_{3} g^{0 j} \delta_{k}^{i} \\
\partial^{i} \partial^{j} G_{k l}^{(1)} & =c_{1}^{\prime \prime} g^{0 i} g^{0 j} g_{k l}+c_{1}^{\prime} g^{i j} g_{k l}+d_{1}^{\prime \prime} g^{0 i} g^{0 j} p_{k} p_{l}+d_{1}^{\prime} g^{i j} p_{k} p_{l} \\
& +d_{1}^{\prime} g^{0 j} \delta_{k}^{i} p_{l}+d_{1}^{\prime} g^{0 j} p_{k} \delta_{l}^{i}+d_{1}^{\prime} g^{0 i} \delta_{k}^{j} p_{l}+d_{1} \delta_{k}^{j} \delta_{l}^{i} \\
& +d_{1}^{\prime} g^{0 i} p_{k} \delta_{l}^{j}+d_{1} \delta_{k}^{i} \delta_{l}^{j} \\
\partial^{i} \partial^{j} G_{(2)}^{k l} & =c_{2}^{\prime \prime} g^{0 i} g^{0 j} g^{k l}+c_{2}^{\prime} g^{i j} g^{k l}+d_{2}^{\prime \prime} g^{0 i} g^{0 j} g^{0 k} g^{0 l}+d_{2}^{\prime} g^{i j} g^{0 k} g^{0 l} \\
& +d_{2}^{\prime} g^{0 j} g^{i k} g^{0 l}+d_{2}^{\prime} g^{0 j} g^{0 k} g^{i l}+d_{2}^{\prime} g^{0 i} g^{j k} g^{0 l}+d_{2} g^{j k} g^{i l} \\
& +d_{2}^{\prime} g^{0 i} g^{0 k} g^{j l}+d_{2} g^{i k} g^{j l} \\
\partial^{i} \partial^{j} G 3_{l}^{k} & =c_{3}^{\prime \prime} g^{0 i} g^{0 j} \delta_{l}^{k}+c_{3}^{\prime} g^{i j} \delta_{l}^{k}+d_{3}^{\prime \prime} g^{0 i} g^{0 j} g^{0 k} p_{l}+d_{3}^{\prime} g^{i j} g^{0 k} p_{l} \\
& +d_{3}^{\prime} g^{0 j} g^{i k} p_{l}+d_{3}^{\prime} g^{0 j} g^{0 k} \delta_{l}^{i}+d_{3}^{\prime} g^{0 i} g^{j k} p_{l}+d_{3} g^{j k} \delta_{l}^{i} \\
& +d_{3}^{\prime} g^{0 i} g^{0 k} \delta_{l}^{j}+d_{3} g^{i k} \delta_{l}^{j} \\
\partial^{i} H_{(1)}^{j k} & =e_{1}^{\prime} g^{0 i} g^{j k}+f_{1}^{\prime} g^{0 i} g^{0 j} g^{0 k}+f_{1} g^{i j} g^{0 k}+f_{1} g^{0 j} g^{i k} \\
\partial^{i} H_{j k}^{(2)} & =e_{2}^{\prime} g^{0 i} g_{j k}+f_{2}^{\prime} g^{0 i} p_{j} p_{k}+f_{2} \delta_{j}^{i} p_{k}+f_{2} p_{j} \delta_{k}^{i} \\
\partial^{i} H 3_{k}^{j} & =e_{3}^{\prime} g^{0 i} \delta_{k}^{j}+f_{3}^{\prime} g^{0 i} g^{0 j} p_{k}+f_{3} g^{i j} p_{k}+f_{3} g^{0 j} \delta_{k}^{i}
\end{aligned}
$$

We get the first order partial derivatives of the $M$-tensor fields $P_{i}{ }^{j h}, Q^{i j}{ }_{h}$, $S_{i j h}, \widetilde{P}_{j}{ }^{i} h, \widetilde{Q}^{i j h}, \widetilde{S}_{i j}{ }^{h}$ with respect to the cotangential coordinates $p_{i}$ and 
we replace these derivatives, and the expressions (17), (18) of the functions $e_{1}, e_{2}, e_{3}, f_{1}, f_{2}, f_{3}$ and of their derivatives in order to obtain the components of the curvature tensor as functions of $a_{1}, a_{2}, a_{3}$ and their derivatives of first, second and third order only. The detailed expressions may be obtained by using the Mathematica package RICCI.

$$
\begin{aligned}
\partial^{i} Q_{h}^{j k} & =\frac{1}{2} \partial^{i} H_{l h}^{(2)}\left(\partial^{j} G_{k l}^{(2)}+\frac{1}{2} H_{l h}^{(2)}\left(\partial^{i} \partial^{j} G_{k l}^{(2)}+\partial^{i} \partial^{k} G_{(2)}^{j l}-\partial^{i} \partial^{l} G_{(2)}^{j k}\right)\right. \\
& +\frac{1}{2} \partial^{i} H 3_{h}^{l}\left(\partial^{j} G 3_{l}^{k}+\partial^{k} G 3_{l}^{k}\right)+\frac{1}{2} H 3_{h}^{l}\left(\partial^{i} \partial^{j} G 3_{l}^{k}+\partial^{i} \partial^{k} G 3_{l}^{k}\right), \\
\partial_{i} \widetilde{Q}^{j k h} & =\frac{1}{2} \partial^{i} H 3_{l}^{h}\left(\partial^{j} G_{(2)}^{k l}+\partial^{k} G_{(2)}^{j l}-\partial^{l} G_{(2)}^{j k}\right) \\
& +\frac{1}{2} H 3_{l}^{h}\left(\partial^{i} \partial^{j} G_{(2)}^{k l}+\partial^{i} \partial^{k} G_{(2)}^{j l}-\partial^{i} \partial^{l} G_{(2)}^{j k}\right) \\
& +\frac{1}{2} \partial^{i} H_{(1)}^{l h}\left(\partial^{j} G 3_{l}^{k}+\partial^{k} G 3_{l}^{j}\right)+\frac{1}{2} H_{(1)}^{l h}\left(\partial^{i} \partial^{j} G 3_{l}^{k}+\partial^{i} \partial^{k} G 3_{l}^{j}\right), \\
\partial^{i} \widetilde{P}_{j}^{k} h & =\frac{1}{2} \partial^{i} H_{l h}^{(2)}\left(\partial^{k} G 3_{j}^{l}-\partial^{l} G 3_{j}^{k}\right)+\frac{1}{2} H_{l h}^{(2)}\left(\partial^{i} \partial^{k} G 3_{j}^{l}-\partial^{i} \partial^{l} G 3_{j}^{k}\right) \\
& +\frac{1}{2} \partial^{i} H 3_{h}^{l}\left(\partial^{k} G_{j l}^{(1)}-R_{m j l}^{0} G_{(2)}^{m k}\right) \\
& +\frac{1}{2} H 3_{h}^{l}\left(\partial^{i} \partial^{k} G_{j l}^{(1)}-R_{m j l}^{i} G_{(2)}^{m k}-R_{m j l}^{0} \partial^{i} G_{(2)}^{m k}\right), \\
\partial^{i} P_{j}^{k h} & =\frac{1}{2} \partial^{i} H 3_{l}^{h}\left(\partial^{k} G 3_{j}^{l}-\partial^{l} G 3_{j}^{k}\right)+\frac{1}{2} H 3_{l}^{h}\left(\partial^{i} \partial^{k} G 3_{j}^{l}-\partial^{i} \partial^{l} G 3_{j}^{k}\right) \\
& +\frac{1}{2} \partial^{i} H_{(1)}^{h l}\left(\partial^{k} G_{j l}^{(1)}-R_{m j l}^{0} G_{(2)}^{m k}\right) \\
& +\frac{1}{2} H_{(1)}^{h l}\left(\partial^{i} \partial^{k} G_{j l}^{(1)}-R_{m j l}^{i} G_{(2)}^{m k}-R_{m j l}^{0} \partial^{i} G_{(2)}^{m k}\right), \\
\partial^{i} S_{j k h} & =\frac{1}{2}\left[\left(c_{2}^{\prime} g^{0 i} R_{m j k}^{0}+c_{2} R_{m j k}^{i}-\partial^{i} \partial^{l} G_{j k}^{(1)}\right) H_{l h}^{(2)}+\left(c_{2} R_{m j k}^{0}-\partial^{l} G_{j k}^{(1)}\right) \partial^{i} H_{l h}^{(2)}\right] \\
& -c_{3}^{\prime} g^{0 i} R_{j k l}^{0} H 3_{h}^{l}-c_{3}\left(R_{j k l}^{i} H 3_{h}^{l}+R_{j k l}^{0} \partial^{i} H 3_{h}^{l}\right), \\
\partial^{i} \widetilde{S}_{j k}^{h} & =\frac{1}{2}\left[\left(c_{2}^{\prime} g^{0 i} R_{m j k}^{0}+c_{2} R_{m j k}^{i}-\partial^{i} \partial^{l} G_{j k}^{(1)}\right) H 3_{l}^{h}+\left(c_{2} R_{m j k}^{0}-\partial^{l} G_{j k}^{(1)}\right) \partial^{i} H 3_{l}^{h}\right] \\
& -c_{3}^{\prime} g^{0 i} R_{j k l}^{0} H_{(1)}^{l h}-c_{3}\left(R_{j k l}^{i} H_{(1)}^{l h}+R_{j k l}^{0} \partial^{i} H_{(1)}^{l h}\right) .
\end{aligned}
$$

The tensor field corresponding to the curvature tensor field of a Kälerian manifold $\left(T^{*} M, G, J\right)$ having constant holomorphic sectional curvature $k$ is given by the formula:

$$
K_{0}(X, Y) Z=\frac{k}{4}[G(Y, Z) X-G(X, Z) Y+G(J Y, Z) J X
$$




$$
-G(J X, Z) J Y+2 G(X, J Y) J Z] .
$$

With respect to the adapted frame $\left\{\delta_{i}, \partial^{j}\right\}_{i, j=1, \ldots, n}$, the expressions are

$$
\begin{aligned}
K_{0}\left(\delta_{i}, \delta_{j}\right) \delta_{k} & =Q Q Q Q_{0}{ }_{i j k}{ }^{h} \delta_{h}+Q Q Q P_{0 i j k h} \partial^{h}, \\
K_{0}\left(\delta_{i}, \delta_{j}\right) \partial^{k} & =Q Q P Q_{0 i j}{ }^{k h} \delta_{h}+Q Q P P_{0 i j}{ }^{k}{ }_{h} \partial^{h}, \\
K_{0}\left(\partial^{i}, \partial^{j}\right) \delta_{k} & =P P Q Q_{0}{ }^{i j}{ }_{k}{ }^{h} \delta_{h}+P P Q P_{0}{ }^{i j}{ }_{k h} \partial^{h}, \\
K_{0}\left(\partial^{i}, \partial^{j}\right) \partial^{k} & =P P P P_{0}{ }^{i j k}{ }_{h} \partial^{h}+P P P Q_{0}{ }^{i j k h} \delta_{h}, \\
K_{0}\left(\partial^{i}, \delta_{j}\right) \delta_{k} & =P Q Q Q_{0}{ }^{i}{ }_{j k}{ }^{h} \delta_{h}+P Q Q P_{0}{ }^{i}{ }_{j k h} \partial^{h}, \\
K_{0}\left(\partial^{i}, \delta_{j}\right) \partial^{k} & =P Q P P_{0}{ }^{i}{ }_{j}{ }_{k}{ }_{h} \partial^{h}+P Q P Q_{0}{ }^{i}{ }_{j}{ }^{k}{ }^{h} \delta_{h},
\end{aligned}
$$

where

$$
\begin{aligned}
Q Q Q Q_{0 i j k}{ }^{h} & =\frac{k}{4}\left[G_{j k}^{(1)} \delta_{i}^{h}-G_{i k}^{(1)} \delta_{j}^{h}-J 3_{i}^{h}\left(J_{j l}^{(1)} G 3_{k}^{l}-J 3_{j}^{l} G_{l k}^{(1)}\right)\right. \\
& \left.+J 3_{j}^{h}\left(J_{i l}^{(1)} G 3_{k}^{l}-J 3_{i}^{l} G_{l k}^{(1)}\right)-2 J 3_{k}^{h}\left(J_{j l}^{(1)} G 3_{i}^{l}-J 3_{j}^{l} G_{i l}^{(1)}\right)\right], \\
Q Q Q P_{0 i j k h} & =\frac{k}{4}\left[J_{i h}^{(1)}\left(J_{j l}^{(1)} G 3_{k}^{l}-J 3_{j}^{l} G_{l k}^{(1)}\right)-J_{j h}^{(1)}\left(J_{i l}^{(1)} G 3_{k}^{l}-J 3_{i}^{l} G_{l k}^{(1)}\right)\right. \\
& \left.+2 J_{k h}^{(1)}\left(J_{j l}^{(1)} G 3_{i}^{l}-J 3_{j}^{l} G_{i l}^{(1)}\right)\right], \\
Q Q P Q_{0 i j}{ }^{k h} & =\frac{k}{4}\left[G 3_{j}^{k} \delta_{i}^{h}-G 3_{i}^{k} \delta_{j}^{h}-J 3_{i}^{h}\left(J_{j l}^{(1)} G_{(2)}^{l k}-J 3_{j}^{l} G 3_{l}^{k}\right)\right. \\
& \left.+J 3_{j}^{h}\left(J_{i l}^{(1)} G_{(2)}^{l k}-J 3_{i}^{l} G 3_{l}^{k}\right)-2 J_{(2)}^{k h}\left(J_{j l}^{(1)} G 3_{i}^{l}-J 3_{j}^{l} G_{i l}^{(1)}\right)\right], \\
Q Q P P_{0 i j}{ }_{k}{ }_{h} & =\frac{k}{4}\left[J_{i h}^{(1)}\left(J_{j l}^{(1)} G_{(2)}^{l k}-J 3_{j}^{l} G 3_{l}^{k}\right)-J_{j h}^{(1)}\left(J_{i l}^{(1)} G_{(2)}^{l k}-J 3_{i}^{l} G 3_{l}^{k}\right)\right. \\
& \left.+2 J 3_{h}^{k}\left(J_{j l}^{(1)} G 3_{i}^{l}-J 3_{j}^{l} G_{i l}^{(1)}\right)\right], \\
P P Q Q_{0}{ }^{i j}{ }_{k}{ }^{h} & =\frac{k}{4}\left[-J_{(2)}^{i h}\left(J 3_{l}^{j} G 3_{k}^{l}-J_{(2)}^{j l} G_{l k}^{(1)}\right)+J_{(2)}^{j h}\left(J 3_{l}^{i} G 3_{k}^{l}-J_{(2)}^{i l} G_{l k}^{(1)}\right)\right. \\
& \left.-2 J 3_{k}^{h}\left(J 3_{l}^{j} G_{(2)}^{i l}-J_{(2)}^{j l} G 3_{l}^{i}\right)\right], \\
P P Q P_{0}{ }^{i j}{ }_{k h} & =\frac{k}{4}\left[G 3_{k}^{j} \delta_{h}^{i} h-G 3_{k}^{i} \delta_{h}^{j}+J 3_{h}^{i}\left(J 3_{l}^{j} G 3_{k}^{l}-J_{(2)}^{j l} G_{l k}^{(1)}\right)\right. \\
& \left.-J 3_{h}^{j}\left(J 3_{l}^{i} G 3_{k}^{l}-J_{(2)}^{i l} G_{l k}^{(1)}\right)+2 J_{k h}^{(1)}\left(J 3_{l}^{j} G_{(2)}^{i l}-J_{(2)}^{j l} G 3_{l}^{i}\right)\right], \\
P P P Q_{0}{ }^{i j k h} & =\frac{k}{4}\left[-J_{(2)}^{i h}\left(J 3_{l}^{j} G_{(2)}^{l k}-J_{(2)}^{j l} G 3_{l}^{k}\right)+J_{(2)}^{j h}\left(J 3_{l}^{i} G_{(2)}^{l k}-J_{(2)}^{i l} G 3_{l}^{k}\right)\right. \\
& \left.-2 J_{(2)}^{k h}\left(J 3_{l}^{j} G_{(2)}^{i l}-J_{(2)}^{j l} G 3_{l}^{i}\right)\right],
\end{aligned}
$$




$$
\begin{aligned}
P P P P_{0}{ }^{i j k}{ }_{h} & =\frac{k}{4}\left[G_{(2)}^{j k} \delta_{h}^{i}-G_{(2)}^{i k} \delta_{h}^{j}+J 3_{h}^{i}\left(J 3_{l}^{j} G_{(2)}^{l k}-J_{(2)}^{j l} G 3_{l}^{k}\right)\right. \\
& \left.-J 3_{h}^{j}\left(J 3_{l}^{i} G_{(2)}^{l k}-J_{(2)}^{i l} G 3_{l}^{k}\right)+2 J 3_{h}^{k}\left(J 3_{l}^{j} G_{(2)}^{i l}-J_{(2)}^{j l} G 3_{l}^{i}\right)\right], \\
P Q Q Q_{0}{ }^{i}{ }_{j k}{ }^{h} & =\frac{k}{4}\left[-G 3_{k}^{i} \delta_{j}^{h}-J_{(2)}^{i h}\left(J_{j l}^{(1)} G 3_{k}^{l}-J 3_{j}^{l} G_{l k}^{(1)}\right)\right. \\
& \left.+J 3_{j}^{h}\left(J 3_{l}^{i} G 3_{k}^{l}-J_{(2)}^{i l} G_{l k}^{(1)}\right)-2 J 3_{k}^{h}\left(J_{j l}^{(1)} G_{(2)}^{i l}-J 3_{j}^{l} G 3_{l}^{i}\right)\right], \\
P Q Q P_{0}{ }^{i}{ }_{j k h} & =\frac{k}{4}\left[G_{j k}^{(1)} \delta_{h}^{i}+J 3_{h}^{i}\left(J_{j l}^{(1)} G 3_{k}^{l}-J 3_{j}^{l} G_{l k}^{(1)}\right)\right. \\
& \left.-J_{j h}^{(1)}\left(J 3_{l}^{i} G 3_{k}^{l}-J_{(2)}^{i l} G_{l k}^{(1)}\right)+2 J_{k h}^{(1)}\left(J_{j l}^{(1)} G_{(2)}^{i l}-J 3_{j}^{l} G 3_{l}^{i}\right)\right], \\
P Q P Q_{0}{ }^{i}{ }_{j}{ }^{k h}{ }^{k} & =\frac{k}{4}\left[-G_{(2)}^{i k} \delta_{j}^{h}-J_{(2)}^{i h}\left(J_{j l}^{(1)} G_{(2)}^{l k}-J 3_{j}^{l} G 3_{l}^{k}\right)\right. \\
& \left.+J 3_{j}^{h}\left(J 3_{l}^{i} G_{(2)}^{l k}-J_{(2)}^{i l} G 3_{l}^{k}\right)-2 J_{(2)}^{k h}\left(J_{j l}^{(1)} G_{(2)}^{i l}-J 3_{j}^{l} G 3_{l}^{i}\right)\right], \\
P Q P P_{0}{ }^{i}{ }_{j}{ }^{k}{ }_{h} & =\frac{k}{4}\left[G 3_{j}^{k} \delta_{h}^{i}+J 3_{h}^{i}\left(J_{j l}^{(1)} G_{(2)}^{l k}-J 3_{j}^{l} G 3_{l}^{k}\right)\right. \\
& \left.-J_{j h}^{(1)}\left(J 3_{l}^{i} G_{(2)}^{l k}-J_{(2)}^{i l} G 3_{l}^{k}\right)+2 J 3_{h}^{k}\left(J_{j l}^{(1)} G_{(2)}^{i l}-J 3_{j}^{l} G 3_{l}^{i}\right)\right] .
\end{aligned}
$$

The Kählerian manifold $\left(T^{*} M, G, J\right)$ is of constant holomorphic sectional curvature if and only if all the components of the difference $K-K_{0}$ vanish. In the study of the vanishing conditions for the components of $K-K_{0}$ we use the following result similar to the lemma 2.1 .

Lemma 3.2. If $\alpha_{1}, \ldots, \alpha_{10}$ are smooth functions on $T^{*} M$ such that

$$
\begin{aligned}
\alpha_{1} g_{h j} g^{i k} & +\alpha_{2} \delta_{h}^{i} \delta_{j}^{k}+\alpha_{3} \delta_{h}^{k} \delta_{j}^{i}+\alpha_{4} g^{i k} p_{h} p_{j}+\alpha_{5} \delta_{j}^{k} p_{h} g^{0 i}+\alpha_{6} \delta_{h}^{k} p_{j} g^{0 i} \\
& +\alpha_{7} \delta_{j}^{i} p_{h} g^{0 k}+\alpha_{8} g_{h j} g^{0 i} g^{0 k}+\alpha_{9} \delta_{h}^{i} p_{j} g^{0 k}+\alpha_{10} p_{h} p_{j} g^{0 i} g^{0 k}=0,
\end{aligned}
$$

then $\alpha_{1}=\cdots=\alpha_{10}=0$.

Proof. If we multiply the expression (20) by $g^{h j} g_{i k}$, we have $\alpha_{1} n^{2}+\alpha_{2} n+\alpha_{3} n+2 \alpha_{4} n t+2 \alpha_{5} t+2 \alpha_{6} t+2 \alpha_{7} t+2 \alpha_{8} n t+2 \alpha_{9} t+4 \alpha_{10} t^{2}=0$.

Since the expression does not depend on the dimension $n$ of the base manifold, we obtain that

$$
\alpha_{1}=0, \alpha_{2}+\alpha_{3}+2\left(\alpha_{4}+\alpha_{8}\right) t=0,\left(\alpha_{5}+\alpha_{6}+\alpha_{7}+\alpha_{9}\right) t+2 \alpha_{10} t^{2}=0 .
$$

Similarly, we get that $\alpha_{2}$ and $\alpha_{3}$ are also zero, if we multiply the expression (20), respectively by $\delta_{i}^{h} \delta_{k}^{j}$ and $\delta_{k}^{h} \delta_{i}^{j}$. 
The product between (20) and $g_{i k} g^{0 h} g^{0 j}, \delta_{k}^{j} g^{0 h} p_{i}, \delta_{k}^{h} g^{0 j} p_{i}, \delta_{i}^{j} g^{0 h} p_{k}$, $g^{h j} p_{i} p_{k}$, or $\delta_{i}^{h} g^{0 j} p_{k}$, leads to some expressions in which the coefficients of $n$ are, respectively $2\left(\alpha_{1} t+2 \alpha_{4} t^{2}\right), 2\left(\alpha_{2} t+2 \alpha_{5} t^{2}\right), 2\left(\alpha_{3} t+2 \alpha_{6} t^{2}\right), 2\left(\alpha_{1} t+2 \alpha_{8} t^{2}\right)$, $2\left(\alpha_{3} t+2 \alpha_{7} t^{2}\right), 2\left(\alpha_{1} t+2 \alpha_{8} t^{2}\right), 2\left(\alpha_{2} t+2 \alpha_{9} t^{2}\right)$. This expressions must vanish for all $t \geq 0$. Since $\alpha_{1}=\alpha_{2}=\alpha_{3}=0$, we obtain that $\alpha_{4}=\cdots=\alpha_{9}=0$ too.

Multiplying by $g^{0 h} g^{0 j} p_{i} p_{k}$, the relation (20) becomes

(21) $4\left[\left(\alpha_{1}+\alpha_{2}+\alpha_{3}\right) t^{2}+2\left(\alpha_{4}+\alpha_{5}+\alpha_{6}+\alpha_{7}+\alpha_{8}+\alpha_{9}\right) t^{3}+4 \alpha_{10} t^{4}\right]=0$.

Taking into account that $\alpha_{i}=0, \forall i=1, \ldots, 9$, it follows from (21) that $\alpha_{10}=0$.

The final theorem gives the condition under which the Kählerian manifold of general natural lift type has constant holomorphic sectional curvature

Theorem 3.3. The Kählerian manifold $\left(T^{*} M, G, J\right)$ with $G$ and $J$ obtained as general natural lifts of the metric $g$ from the Riemannian manifold $(M, g)$, has constant holomorphic sectional curvature $k$ if and only if the parameter $\lambda$ is given by

$$
\lambda=\frac{4 a_{1} c}{k\left(a_{1}^{2}+2 c t+2 a_{3}^{2} c t\right)}
$$

Proof. The expressions of the differences that we study are quite long, but in $P Q P P^{i}{ }_{j}{ }_{h}-P Q P P_{0}{ }_{j}{ }_{j}{ }_{h}$ two coefficients have shorter expressions. From the first term, which contains $g_{h j} g^{i k}$, by imposing the vanishing condition for the coefficient, we get

$$
\lambda^{\prime}=-\lambda \frac{a_{1}^{\prime}\left(a_{1}^{2}-2 c t-2 a_{3}^{2} c t\right)+2 a_{1} c\left(1+a_{3}^{2}+2 a_{3} a_{3}^{\prime} t\right)}{a_{1}\left(a_{1}^{2}+2 c t+2 a_{3}^{2} c t\right)},
$$

If we substitute this expression in the second term (which contains $\delta_{h}^{i} \delta_{j}^{k}$ ) we have that $\lambda$ is given by (22).

The value of $\lambda^{\prime}$ obtained by differentiating the relation (22), coincides with that obtained by replacing $\lambda$ in (23). Using RICCI, we prove that all the components of the difference $K-K_{0}$ are zero, when the obtained values of $\lambda^{\prime}, \lambda^{\prime \prime}$ and $\lambda^{\prime \prime \prime}$ are replaced in these components. The computation of some differences, such as $P Q P P^{i}{ }_{j}{ }_{h}-P Q P P_{0}{ }_{j}{ }_{j}{ }_{h}, P Q P Q^{i}{ }_{j}{ }^{k h}-$ $P Q P Q_{0}{ }_{j}{ }_{j}^{k h}, P Q Q P^{i}{ }_{j k h}-P Q Q P_{0}{ }_{j k h}$, and $P Q Q Q^{i}{ }_{j k}{ }^{h}-P Q Q Q_{0}{ }^{i}{ }_{j k}{ }^{h}$ is quite hard, since after imposing the integrability conditions for the almost 
complex structure $J$, the expressions become very long, and the command TensorSimplify did not work on a PC with a RAM memory of $2 \mathrm{~GB}$. Thus I had to impose the integrability conditions in every coefficient appearing in the above differences, and to sum the expressions afterwards.

Remark. If $a_{3}=0$ we obtain the condition for $\left(T^{*} M, G, J\right)$ to have constant holomorphic sectional curvature in the case where $G, J$ are natural lifts of diagonal type (see [18]).

Acknowledgements. The author wants to express acknowledgements to Professor Oproiu for the techniques learned during the elaboration of the joint work [4], for the suggestions, encouragements, and support throughout the present work and throughout the $\mathrm{PhD}$ period.

\section{REFERENCES}

1. Anastasiei, M. - Locally conformal Kaehler structures on tangent manifold of a space form, Libertas Math., 19 (1999), 71-76.

2. BucĂtaru, I.; Miron, R. - Finsler-Lagrange Geometry. Applications to Dynamical Systems, Editura Academiei Române, Bucharest, 2007.

3. Druţă, S.L. - Cotangent bundles with general natural Kähler structures, Rev. Roumaine Math. Pures Appl., 54 (2009), 13-23.

4. Druţă, S.; Oproiu, V. - General natural Kähler structures of constant holomorphic sectional curvature on tangent bundles, An. Ştiinţ. Univ. "Al. I. Cuza" Iaşi. Mat. (N.S.), 53 (2007),149-166.

5. Kolár̆, I.; Michor, P.; Slovák, J. - Natural Operations in Differential Geometry, Springer-Verlag, Berlin, 1993.

6. Kowalski, O.; Sekizawa, M. - Natural transformations of Riemannian metrics on manifolds to metrics on tangent bundles. A classification, Bull. Tokyo Gakugei Univ., 40 (1988), 1-29.

7. Miron, R.; Matsumoto, K. - The almost Hermitian structures determined by the Riemannian structures on the tangent bundle, Finsler and Lagrange geometries (Iasi, 2001), 119-127, Kluwer Acad. Publ., Dordrecht, 2003.

8. Mok, K.P.; Patterson, E.M.; Wong, Y.C. - Structure of symmetric tensors of type $(0,2)$ and tensors of type $(1,1)$ on the tangent bundle, Trans. Amer. Math. Soc., 234 (1977), 253-278. 
9. Munteanu, M.I. - CR-structures on the unit cotangent bundle and Bochner type tensor, An. Ştiinţ. Univ. "Al. I. Cuza" Iaşi, Mat. (N.S.), 44 (1998), 125-136.

10. Munteanu, M.I. - Cheeger Gromoll type metrics on the tangent bundle, Sci. Ann. Univ. Agric. Sci. Vet. Med., 49 (2006), 257-268.

11. Munteanu, M.I. - Old and new structures on the tangent bundle, Proceedings of the Eighth International Conference on Geometry, Integrability and Quantization, June 9-14, 2006, Varna, Bulgaria, Ed. I.M. Mladenov and M. de Leon, Sofia 2007, 264-278.

12. Munteanu, M.I. - Some aspects on the geometry of the tangent bundles and tangent sphere bundles of a Riemannian manifold, Mediterr. J. Math., 5 (2008), 43-59.

13. Oproiu, V. - A generalization of natural almost Hermitian structures on the tangent bundles, Math. J. Toyama Univ., 22 (1999), 1-14.

14. Oproiu, V. - Some new geometric structures on the tangent bundles, Publ. Math. Debrecen, 55 (1999), 261-281.

15. Oproiu, V.; PApaghiuc, N. - A pseudo-Riemannian structure on the cotangent bundle, An. Şt. Univ. "Al. I. Cuza" Iaşi, 36 (1990), 265-276.

16. Oproiu, V.; PApaghiuc, N. - Another pseudo-Riemannian structure on the cotangent bundle, Bul. Inst. Politehn. Iaşi, 37 (1991), 27-33.

17. Oproiu, V.; Papaghiuc, N. - Locally symmetric cotangent bundles, Mat. Vesnik, 42 (1990), 221-232.

18. Oproiu, V.; Poroşniuc, D.D. - A Kähler Einstein structure on the cotangent bundle of a Riemannian manifold, An. Ştiinţ. Univ. "Al. I. Cuza" Iaşi. Mat. (N.S.), 49 (2003), 399-414.

19. Poroşniuc, D.D. - A class of locally symmetric Kähler Einstein structures on the nonzero cotangent bundle of a space form, Balkan J. Geom. Appl., 9 (2004), 68-81.

20. Poroşniuc, D.D. - A Kähler Einstein structure on the nonzero cotangent bundle of a space form, Ital. J. Pure Appl. Math., 18 (2005), 223-234.

21. Poroşniuc, D.D. - A locally symmetric Kähler Einstein structure on the cotangent bundle of a space form, Balkan J. Geom. Appl., 9 (2004), 87-95.

22. Poroşniuc, D.D. - A locally symmetric Kähler Einstein structure on a tube in the nonzero cotangent bundle of a space form, An. Ştiinţ. Univ. "Al.I. Cuza" Iaşi. Mat. (N.S.), 50 (2004), 315-326.

23. Yano, K.; Ishinara, S. - Tangent and Cotangent Bundles: Differential Geometry, Pure and Applied Mathematics, Marcel Dekker, Inc., New York, 1973. 